\title{
REVIEW
}

\section{Clinical review: Consensus recommendations on measurement of blood glucose and reporting glycemic control in critically ill adults}

\author{
Simon Finfer', Jan Wernerman², Jean-Charles Preiser³, Tony Cass' ${ }^{4}$, Thomas Desaive ${ }^{5}$, Roman Hovorka ${ }^{6}$, \\ Jeffrey I Joseph7, Mikhail Kosiborod ${ }^{8}$, James Krinsley ${ }^{9}$, lain Mackenzie ${ }^{10}$, Dieter Mesotten ${ }^{11}$, Marcus J Schultz ${ }^{12}$, \\ Mitchell G Scott ${ }^{13}$, Robbert Slingerland ${ }^{14}$, Greet Van den Berghe ${ }^{11}$ and Tom Van Herpe ${ }^{11,15}$
}

\begin{abstract}
The management reporting and assessment of glycemic control lacks standardization. The use of different methods to measure the blood glucose concentration and to report the performance of insulin treatment yields major disparities and complicates the interpretation and comparison of clinical trials. We convened a meeting of 16 experts plus invited observers from industry to discuss and where possible reach consensus on the most appropriate methods to measure and monitor blood glucose in critically ill patients and on how glycemic control should be assessed and reported. Where consensus could not be reached, recommendations on further research and data needed to reach consensus in the future were suggested. Recognizing their clear conflict of interest, industry observers played no role in developing the consensus or recommendations from the meeting. Consensus recommendations were agreed for the measurement and reporting of glycemic control in clinical trials and for the measurement of blood glucose in clinical practice. Recommendations covered the following areas: How should we measure and report glucose control when intermittent blood glucose measurements are used? What are the appropriate performance standards for intermittent blood glucose monitors in the ICU? Continuous or automated intermittent glucose monitoring - methods and technology: can we use the same measures for assessment of glucose control with continuous and intermittent monitoring? What is acceptable performance for continuous glucose monitoring systems? If implemented, these recommendations have the potential to minimize the discrepancies in the conduct and reporting of clinical trials and to improve glucose control in clinical practice. Furthermore, to be fit for use, glucose meters and continuous monitoring systems must match their performance to fit the needs of patients and clinicians in the intensive care setting.
\end{abstract}

\section{Background}

In 2001 Van den Berghe and colleagues reported that targeting a blood glucose concentration of 4.4 to $6.1 \mathrm{mmol} / \mathrm{l}(80$ to $110 \mathrm{mg} / \mathrm{dl})$ in adult patients treated in a surgical ICU reduced both morbidity and mortality [1]. This research had a profound effect on the way the management of blood glucose in critically ill patients was perceived, with widespread recognition that blood glucose management could affect important patient outcomes. Van den Berghe and colleagues' paper led to a large body

*Correspondence: Jean-charles.preiser@erasme.ulb.ac.be

${ }^{3}$ Department of Intensive Care, Erasme University Hospital, 808 route de Lennik,

B-1070 Brussels, Belgium

Full list of author information is available at the end of the article of research from numerous investigators that produced a much better understanding of the issues surrounding the control of blood glucose, also highlighting areas where additional research and agreement are urgently needed.

Completed and ongoing research has highlighted two critically important issues that currently hamper our understanding of the best approach to glycemic control in critically ill patients. The first relates to how blood glucose concentration is measured, because inaccurate blood glucose measurement adversely effects glycemic control and may result in direct harm to patients. The second issue relates to how glycemic control is reported currently this is not standardized, which makes comparing the results of much clinical research impossible [2-5].

In Van den Berghe and colleagues' original trial, blood glucose concentration was measured using a blood gas 
analyzer, a method that has been shown to be accurate [2]. Subsequent trials have used a variety of methods to measure the blood glucose concentration, which have included single specified bedside glucose meters [6,7] or combined methods using specified or unspecified glucose meters and blood gas analyzers variably both within and between sites in multicenter trials $[8,9]$. With increasing awareness of the association between hyperglycemia [10], hypoglycemia [11], and glucose variability [12] with increased mortality and morbidity has come the realization that more accurate measurement of blood glucose concentration might be crucial for improving patients' outcomes. Accurate measurement of the blood glucose concentration requires not only the use of accurate analyzers but also standardized blood sampling and handling. Pre-analytical errors arise due to variable blood sampling and handling, and these errors may be larger than those arising from the use of inaccurate analyzers.

Point-of-care devices were not designed for ICU use or to be used to regulate insulin infusions in critically ill patients. The ICU is a unique environment where readings from glucose meters may be subject to further inaccuracies due to rapid changes in hematocrit or oxygenation and interference from medications and other physical or chemical factors [13]. In response to these concerns, newer glucose meters try to correct known problems and monitors capable of continuous or automated intermittent measurement of blood glucose are being developed specifically for use in the ICU.

A further issue is the assessment and reporting of glycemic control [3]. Glycemic control can be reported in many different ways with a focus on different measures of central tendency (mean, median, mode, and so forth) and dispersion (standard deviation, range, interquartile range). Within these domains the dimension of time also has to be considered along with the impact of the frequency with which blood glucose concentration is measured. This consideration has resulted in glycemic control being reported in many different ways, which introduces further difficulty when trying to assess the adequacy of glycemic control and to understand and to compare the results of the various clinical trials.

In light of the uncertainties in this area we convened a meeting of interested parties and experts plus invited observers from industry to discuss and where possible reach consensus on the most appropriate methods to measure and monitor blood glucose in critically ill patients and on how glycemic control should be assessed and reported. Recognizing their clear conflict of interest, industry observers played no role in developing the consensus or recommendations from the meeting. Where the academic participants could not reach consensus, they sought to make recommendations on further research and data needed to reach consensus in the future.
The following issues were addressed: How should we measure and report glucose control when intermittent blood glucose measurements are used? What are the appropriate performance standards for intermittent blood glucose monitors in the ICU? Continuous or automated intermittent glucose monitoring - methods and technology: can we use the same measures for assessment of glucose control with continuous and intermittent monitoring? What is acceptable performance for continuous glucose monitoring systems?

\section{How should we measure and report glucose control when intermittent blood glucose measurements are used?}

Glycemic control is commonly reported using measures of central tendency and dispersion, and additionally most investigators report either the number of patients who experience hypoglycemia and/or the number of occasions on which hypoglycemia occurs. Some investigators have reported the time to attain the target blood glucose and the proportion of time blood glucose concentration is within the target range. Additionally, a variety of more complex measures have been proposed that assess elements of glycemic control, such as time and distance away from a target range and the degree of variability in blood glucose concentration [3,14]. Mackenzie and colleagues analyzed data from four adult ICUs in one hospital in the UK and concluded that each of the various metrics was measuring one of three features of glycemic control [3]. Using cluster analysis they classified 13 reported metrics into two families representing central tendency and dispersion, or measures of minimum glucose that were related to neither family. Each of these three features of glycemic control (central tendency, dispersion and minimum glucose) had an association with patients' outcomes (Figure 1).

The group then considered the effect of measurement frequency and duration of monitoring on the various measures of glycemic control. With regard to simple measures it is clear that all indices may be affected by frequency of measurement and duration of monitoring. For example, a reported mean blood glucose that just averages all measurements will be lower in a study that mandates frequent measurements around the lower limit of normal when compared with another protocol that does not. Likewise, different durations of treatment and study may produce different summary results as all indices tend to improve over 3 to 4 days and then stabilize [15].

A further consideration discussed was how summary measures for populations of patients should be calculated and reported. For example, a mean blood glucose concentration for a population over time could represent the sum of all the blood glucose measures of that population 


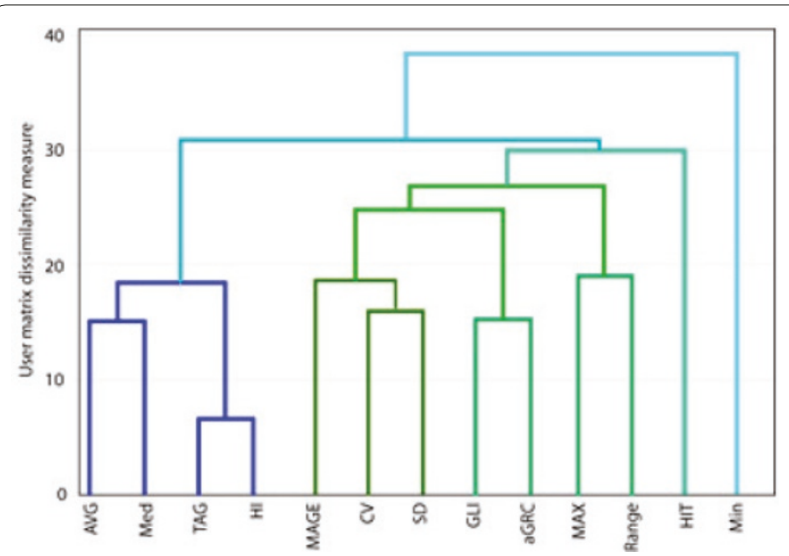

Figure 1. Dendrogram of measures of central tendency, dispersion and hypoglycemia. See [3] for a full explanation. Reproduced with permission.

divided by the number of measures. An alternative would be to calculate each patient's mean blood glucose concentration (the sum of all that patient's blood glucose measures divided by the number of measures) and then report the mean of the individual patients' mean blood glucose concentrations. These might be quite different numbers depending on how many measurements each patient contributed. Similar considerations apply to other measures such as the standard deviation, which may be reported as the standard deviation of the individual glucose measurement or the standard deviation of the individual patient means. Although not commonly reported it is critical that studies explain how such summary measures are calculated.

\section{Meeting consensus}

Given that many measures are effectively reporting similar variables, the consensus was that simple measures which are intuitively understood should be reported and standardized as the appropriate measures of glycemic control.

Trials or observational studies should report the following:

1. Central tendency - for blood glucose concentration measurements from a population of patients, the median and interquartile range of individual patient means should be reported.

2. Dispersion - one should calculate the standard deviation of blood glucose concentration for each patient, and then report the median and interquartile range of standard deviations for the population.

3. Hypoglycemia - as a minimum, investigators should report the number and percentage of patients experiencing at least one episode of severe and moderate hypoglycemia. Severe hypoglycemia is defined as blood glucose concentration $\leq 2.2 \mathrm{mmol} / \mathrm{l}(\leq 40 \mathrm{mg} / \mathrm{dl})$; moderate hypoglycemia is defined as a blood glucose concentration of 2.3 to $3.9 \mathrm{mmol} / \mathrm{l}$ (41 to $70 \mathrm{mg} / \mathrm{dl}$ ). The number and percentage of patients experiencing hypoglycemia related to insulin treatment (iatrogenic) and unrelated to insulin treatment (spontaneous; for example, terminal event in patient dying of hepatic failure) should be reported separately. Investigators are strongly encouraged to report as much detail as possible on hypoglycemia events (for instance, duration, associated symptoms, amount of glucose administered, next blood glucose), if necessary in an electronic supplement, as there will be no randomized trials of hypoglycemia and retrospective or registry studies often have only sparse information.

The recommendation to report the above metrics was a pragmatic one, with the group recognizing that further research was needed to define the optimal metric in each of the domains. The optimal metric would be the one with the best balance of simplicity and performance in terms of association with and prediction of clinical outcome.

In addition to reporting the above metrics, investigators should also report the frequency of blood glucose measurement, the duration of monitoring, the nature of blood sampling (that is, capillary vs. peripheral venous vs. central venous vs. arterial sampling) and the technology used to measure blood glucose.

\section{What are the appropriate performance standards for intermittent blood glucose monitors in the ICU?} Measurement of blood glucose concentration in ICUs is currently performed almost entirely intermittently, with analysis using either point-of-care glucose meters or blood gas analyzers. Although accurate data are not available, most measurements are probably made on glucose meters and the majority of samples are capillary blood obtained by finger pricks. The use of glucose meters and sampling capillary blood both have the potential to introduce errors into the measurement of blood glucose concentration.

The accuracy of glucose meters has been the subject of a number of studies, with the near-universal conclusion that they are not sufficiently accurate for use in the ICU $[2,4,5,16]$. Current regulatory and consensus guidelines for glucose meter accuracy are dated and were developed primarily for self-monitoring home-use devices. For instance, the current International Standards Organization standard (ISO 15197) requires that $95 \%$ of results from a glucose meter be within $20 \%$ (or $15 \mathrm{mg} / \mathrm{dl}$ for values $<75 \mathrm{mg} / \mathrm{dl}$ ) of results obtained from a reference measurement such as a central laboratory hexokinase method or a Yellow Springs Instrument (Yellow Springs Instruments, Yellow Springs, OH, USA). Similarly, the Clinical Laboratory and Standards Institute (CLSI C30-A2) states that $95 \%$ of meter values must be within $20 \%$ (or $15 \mathrm{mg} / \mathrm{dl}$ for values $<75 \mathrm{mg} / \mathrm{dl}$ ) of a reference method. In the USA, 
the US Food and Drug Administration has used similar criteria to approve glucose meters for marketing. One obvious problem with these criteria is that they allow 1 in $20(5 \%)$ meter readings to differ by any amount from the reference method, which could lead to dangerous changes in insulin therapy in critically ill patients.

Furthermore, many current meters are susceptible to interferences from reducing substances such as ascorbic acid and acetaminophen (paracetamol) and many are still affected by the patient's hematocrit $[13,17]$. The effect of hematocrit is particularly concerning in critical care where a patient with a true glucose of $80 \mathrm{mg} / \mathrm{dl}$ and a hematocrit of 0.25 may have a positive bias of as much as $18 \mathrm{mg} / \mathrm{dl}$ [17]. In addition, meters are subject to operator error, which is difficult to quantify but occurred in 0.5 to $0.8 \%$ of measurements at one institution [5]. Despite being in common use in critical care units, numerous modeling studies have shown that current meter precision and accuracy are inadequate to avoid clinically significant errors in insulin dosing that might induce hypoglycemia $[18,19]$. These studies also show that 4 to $15 \%$ of hypoglycemic events may be undetected by current meters. Interestingly, these modeling studies all suggest that a total analytic error $((2 \times \%$ Coefficient of variation) $+\%$ Bias) of 10 to $15 \%$ will avoid most dosing errors and undetected hypoglycemia $[18,19]$.

In 1987 Clarke and colleagues described an error grid that could be used to describe the clinical accuracy of systems designed for the self-monitoring of blood glucose concentration by patients with diabetes and also indicated when inaccuracies in the measurement were likely to result in treatment errors that were potentially dangerous for patients [20]. While the original error grid may have fulfilled its initial purpose, its design is based on assumptions that limit its value in the management of today's critically ill patients. The grid was designed to be used with a target range of 70 to $180 \mathrm{mg} / \mathrm{dl}$ and assumes no change in treatment when readings lie within that range. The grid assumes that treatment of blood glucose concentration outside the target range will be similar to that of the patients treated in Clarke's institution in 1987. Furthermore, the grid makes no allowance for the rate at which blood glucose concentration is changing or the frequency with which the blood glucose concentration is being measured [20]. The assumptions underlying the design of the error grid do not hold true in critically ill patients and make the original Clarke and colleagues error grid unsuitable for use in critically ill patients. While modified error grids have been described, their value in critically ill patients is as yet unproven [21].

Alternatives to the use of glucose meters are measurement in the hospital's central laboratory or using a blood gas analyzer in the ICU. Accuracy standards for measurement of blood glucose in hospital laboratories are $\pm 6 \mathrm{mg} /$ $\mathrm{dl}(0.33 \mathrm{mmol} / \mathrm{l})$ or $10 \%$ (whichever is greater) in the USA [22], $\pm 9.4 \%$ in the Netherlands [23], and $\pm 0.4 \mathrm{mmol} / \mathrm{l}$ (or $\pm 8 \%$ above $5 \mathrm{mmol} / \mathrm{l}$ ) in Australia [24]. Although central laboratory measurement is much more accurate, the time delay in sending samples to the laboratory makes this an impractical solution for the ICUs in most hospitals. A more practical solution, but one that may have considerable cost implications, is to measure the blood glucose concentration in a blood gas analyzer because the majority of ICUs in the developed world will have such an analyzer in the ICU. Measurements from a properly maintained blood gas analyzer will have similar accuracy to central laboratory measurements [2].

\section{Sampling site}

An additional consideration is that the blood glucose concentration varies in different vascular beds and the site from which blood is sampled can introduce further errors. The blood glucose concentration in radial arterial blood will be approximately $0.2 \mathrm{mmol} / \mathrm{l}$ higher than that in blood sampled from a peripheral vein, and 0.3 to $0.4 \mathrm{mmol} / \mathrm{l}$ higher than that in blood sampled from the superior vena cava. Sampling capillary blood in ICU patients, particularly in those who are hemodynamically unstable and being treated with vasopressors, can introduce large errors when compared with a reference method in which glucose is measured in central venous or arterial samples [2,25].

The frequency with which the blood glucose concentration is measured in the ICU makes venipuncture impractical, and viable alternatives are to sample from indwelling arterial or venous catheters. Sampling from indwelling vascular catheters may increase the risk of catheter-related bloodstream infection but this risk has not been quantified. Obviously, when sampling from indwelling catheters it is essential to avoid contamination from infusions of glucose-containing fluids. This caution is particularly important with venous catheters, but accidental use of $5 \%$ glucose in an arterial-line flush bag has resulted in the death of at least one patient [26]. A further potential drawback to sampling from indwelling catheters is the discarding of large volumes of blood to obtain uncontaminated samples. In the case of arterial catheters there is also the potential for contamination by the flush solution if an inadequate volume of dead space blood is withdrawn. These concerns can be reduced or obviated by using a blood conservation system, but this adds to the cost of the procedure.

\section{Recommendations for clinical practice and for conducting and reporting clinical trials and observational studies (Table 1)}

There was general consensus that the current International Standards Organization standard that was developed specifically for home-use meters is not 


\section{Table 1. Summary of the recommendations}

\section{Blood sampling}

1. Patients whose severity of illness justifies invasive vascular monitoring

a. All blood samples should be drawn from an arterial line

b. If an arterial line is temporarily or permanently unavailable, sample from a venous line

c. Capillary (needle stick) samples are inaccurate and should not be used

2. Patients whose severity of illness does not justify invasive vascular monitoring

a. Capillary (needle stick) samples may be used

3. Clinical research papers should report the number and percentage of blood samples obtained from arterial catheters, central and peripheral venous catheters and capillary (needle stick) samples

Choice of blood glucose analyzer in clinical research in critical care units

a. Samples taken from arterial or central venous catheters should be analyzed in a central laboratory or blood gas analyzer; a blood gas analyzer should be the default analyzer

b. Only when capillary samples are taken from patients without invasive vascular monitoring is analysis using a glucose meter acceptable

c. Clinical research papers should report the number and percentage of samples analyzed using central laboratory or blood gas analyzers or glucose meters. In all cases, the make and model of the analyzer used should be reported along with routine calibration and quality assurance measures

Reporting glycemic control - trials or observational studies should report

1. Central tendency - for blood glucose concentration measurements from a population of patients, the median and interquartile range of individual patient means should be reported

2. Dispersion - calculate the standard deviation of blood glucose concentration for each patient then report the median and interquartile range of standard deviations for the population

3. Hypoglycemia - as a minimum, investigators should report the number and percentage of patients experiencing at least one episode of severe and moderate hypoglycemia (blood glucose concentration $\leq 2.2$ ( $\leq 40 \mathrm{mg} / \mathrm{dl}$ ) and 2.3 to $3.9 \mathrm{mmol} / \mathrm{l}$ (41 to $70 \mathrm{mg} / \mathrm{dl}$ ) respectively). Report separately the number and percentage of patients experiencing hypoglycemia related to insulin treatment (iatrogenic) and unrelated to insulin treatment (spontaneous)

For severe hypoglycemia, report duration of hypoglycemia, associated symptoms, amount of glucose administered, and next blood glucose concentration

\section{Appropriate standards for intermittent measurement of blood glucose in the ICU}

Blood sampling

1. Patients whose severity of illness justifies invasive vascular monitoring

a. All blood samples should be drawn from an arterial line

b. If an arterial line is temporarily or permanently unavailable, sample from a venous line

c. Capillary (needle stick) samples are inaccurate and should not be used

2. Patients whose severity of illness does not justify invasive vascular monitoring

a. Capillary (needle stick) samples may be used

Choice of blood glucose analyzer in clinical practice in critical care units

1. Patients whose severity of illness justifies invasive vascular monitoring

a. Samples taken from arterial or central venous catheters should be analyzed in a central laboratory or blood gas analyzer; a blood gas analyzer should be the default analyzer, central laboratory measurements should only be used if results can be obtained without delay

2. Patients whose severity of illness does not justify invasive vascular monitoring

a. Only when capillary samples are taken from patients considered well to need invasive vascular monitoring is analysis using a glucose meter acceptable

Accuracy of blood glucose analyzers used in clinical practice in critical care units

1. Patients whose severity of illness justifies invasive vascular monitoring

a. Central laboratory analyzers and blood gas analyzers in the ICU should perform to currently acceptable international standards (for example, $\pm 0.4 \mathrm{mmol} / \mathrm{l}($ or $\pm 8 \%$ above $5 \mathrm{mmol} / \mathrm{l})$ )

2. Patients whose severity of illness does not justify invasive vascular monitoring

a. The minimum standard for glucose meters to be used in critically ill patients should be that $98 \%$ of readings are within $12.5 \%$ of a reference standard (or within $0.55 \mathrm{mmol} / \mathrm{l}$ for readings $<5.5 \mathrm{mmol} / \mathrm{l}$ ). The remaining $2 \%$ of readings should be within $20 \%$ of a reference standard

\section{Continuous and automated intermittent blood glucose monitoring}

Assessment of glycemic control when continuous or automated intermittent blood glucose monitoring used

1. Currently there are few data to guide the choice of appropriate metrics for reporting glycemic control when continuous or automated intermittent blood glucose monitoring is used. There is a need to define measures that are associated with important patient-centered outcomes such as mortality and major morbidity

Comparing glycemic control with continuous versus intermittent measurement of blood glucose

1. Comparison of the glycemic control achieved with continuous versus intermittent monitoring must be evaluated in randomized controlled trials with both groups of patients having a continuous monitor but the output from the continuous monitor masked in the control group where blood glucose is managed by intermittent monitoring 


\section{Table 1. Continued}

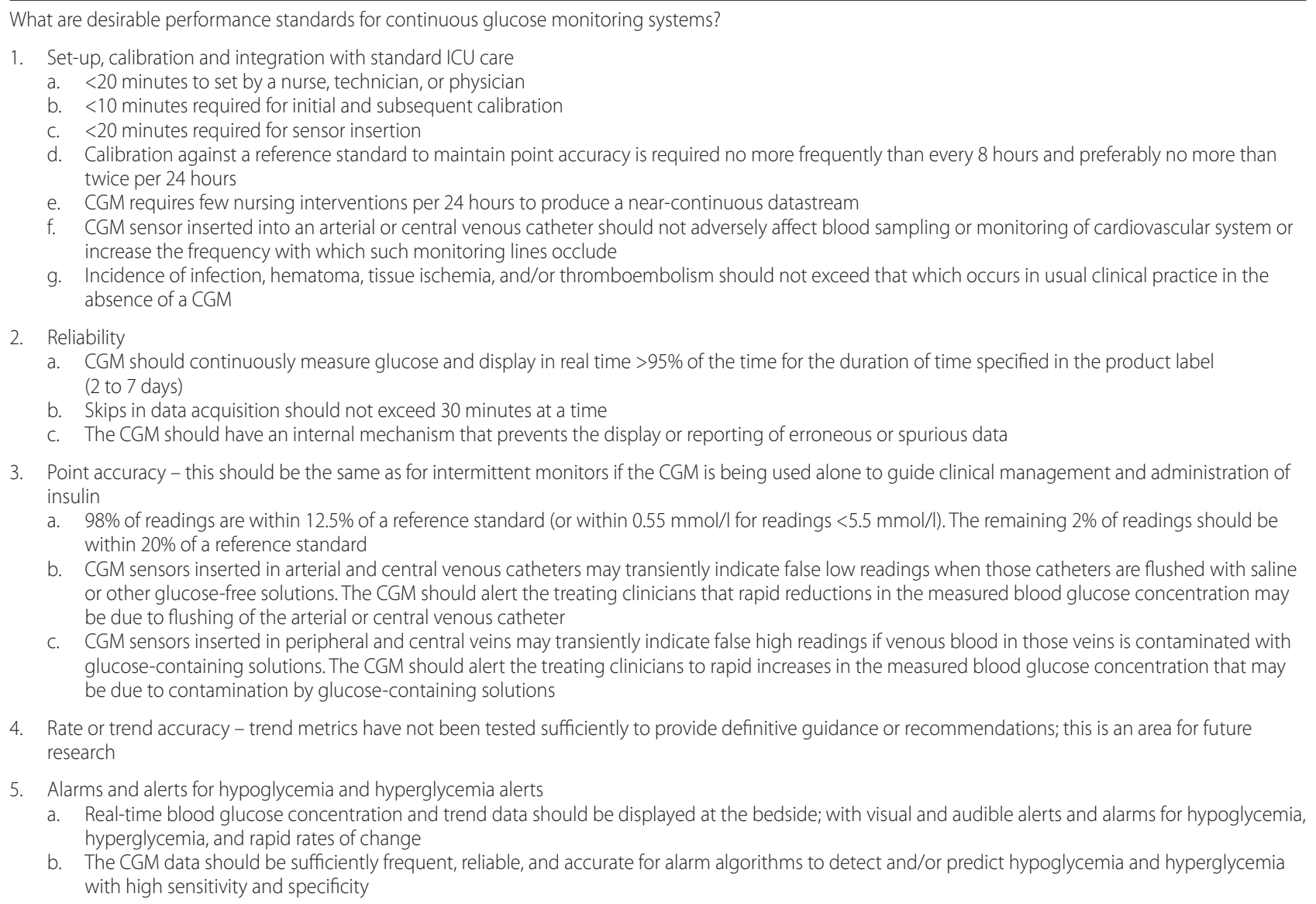

Recommendations for conducting and reporting clinical trials and observational studies. CGM, continuous or automated intermittent glucose monitoring system.

appropriate for the measurement of the blood glucose concentration in critically ill patients and that sampling of capillary blood introduces unacceptable errors and uncertainty. The meeting also recognized that illness severity and case mix can vary greatly between individual units and countries, and that any recommendations should consider these factors. For example, it may be appropriate to target moderately tight glucose control in patients in a cardiac care unit but such patients would not have indwelling arterial catheters or central venous catheters.

Recommendations for clinical practice are as follows:

1. Blood sampling for glucose measurement in critical care:

a. All patients whose severity of illness justifies the presence of invasive vascular monitoring (an indwelling arterial and/or central venous catheter) should have all samples for measurement of the blood glucose concentration taken from the arterial catheter as the first option. If blood cannot be sampled from an arterial catheter or an arterial catheter is temporarily or permanently unavailable, blood may be sampled from a venous catheter as a second option; appropriate attention must be paid to maintaining sterility and avoiding contamination of the sample by flush solution.

b. Only when a patient's severity of illness does not justify the presence of invasive vascular monitoring are capillary samples acceptable for the measurement of the blood glucose concentration.

2. Choice of blood glucose analyzer in clinical practice in critical care units:

a. Samples taken from arterial or central venous catheters should be analyzed in a central laboratory or blood gas analyzer. For most ICUs the delay associated with central laboratory analysis will be unacceptable and therefore a blood gas analyzer should be the default analyzer.

b. Only when capillary samples are taken from patients considered too well to need invasive vascular monitoring is analysis using a glucose meter acceptable.

3. Accuracy of blood glucose analyzers used in clinical practice in critical care units:

a. Central laboratory analyzers and blood gas analyzers in the ICU should perform to currently acceptable international standards - accuracy 
standards for measurement of blood glucose in hospital laboratories are $\pm 6 \mathrm{mg} / \mathrm{dl}(0.33 \mathrm{mmol} / \mathrm{l})$ or $10 \%$ (whichever is greater) in the USA [22], $\pm 9.4 \%$ in the Netherlands [23], and $\pm 0.4 \mathrm{mmol} / \mathrm{l}$ (or $\pm 8 \%$ above $5 \mathrm{mmol} / \mathrm{l}$ ) in Australia [24].

b. The current International Standards Organization standard for glucose meters is not appropriate for critically ill patients - the minimum standard for glucose meters to be used in critically ill patients should be that $98 \%$ of readings are within $12.5 \%$ of a reference standard (or within $0.55 \mathrm{mmol} / \mathrm{l}$ for readings $<5.5 \mathrm{mmol} / \mathrm{l}$ ); the remaining $2 \%$ of readings should be within $20 \%$ of a reference standard.

Recommendations for conducting and reporting clinical trials and observational studies are as follows:

1. Site of blood sampling for glucose measurement in clinical research:

a. All patients whose severity of illness justifies the presence of invasive vascular monitoring (an indwelling arterial and/or central venous catheter) should have all samples for measurement of blood glucose concentration taken from the arterial catheter as the first option. If blood cannot be sampled from an arterial catheter or an arterial catheter is temporarily or permanently unavailable, blood may be sampled from a venous catheter as a second option; appropriate attention must be paid to maintaining sterility and avoiding contamination of the sample by flush solution.

b. Only when a patient's severity of illness does not justify the presence of invasive vascular monitoring are capillary samples acceptable for the measurement of blood glucose concentration.

c. Clinical research papers should report the number and percentage of blood samples obtained from arterial catheters, central and peripheral venous catheters and capillary (needle sticks) samples.

2. Choice of blood glucose analyzer in clinical research in critical care units:

a. Samples taken from arterial or central venous catheters should be analyzed in a central laboratory or blood gas analyzer. For most ICUs the delay associated with central laboratory analysis will be unacceptable and therefore a blood gas analyzer should be the default analyzer.

b. Only when capillary samples are taken from patients without invasive vascular monitoring is analysis using a glucose meter acceptable.

c. Clinical research papers should report the number and percentage of samples analyzed using central laboratory or blood gas analyzers or glucose meters. In all cases, the make and model of the analyzer used should be reported along with routine calibration and quality assurance measures.

\section{Continuous glucose monitoring - methods and technology}

More than 30 years of intense academic and commercial activity in the development of continuous glucose monitoring systems have been focused to a great degree on ambulatory measurements in patients with type I diabetes. The technologies developed to date encompass both electrochemical and optical measurement principles using catalytic (enzyme-based) and binding modes. Unlike in the ICU, a major emphasis in ambulatory medicine has been on minimally or non-invasive measurements with tissue probes rather than catheter-based sensors. Most ambulatory systems have measured glucose in the interstitial space with regular calibration against a reference blood sample, often a capillary sample.

Numerous techniques are available for continuous glucose monitoring in the ICU, including microdialysis and optical methods such as absorption spectroscopy, optical scattering and fluorescence [27]. The blood glucose concentration can be measured in vitro by sensors that sit in the vascular or interstitial space or $e x$ vivo by drawing blood samples or a dialysate to a sensor from an indwelling vascular catheter or dialysis membrane. Systems that intermittently draw blood to an externally based sensor may be described as automated intermittent monitors rather than continuous glucose monitors. Potential advantages of continuous glucose monitors include the ability to observe trends in blood glucose concentration and to intervene before the blood glucose concentration enters an unacceptable range, and removal of operator error both in the timing of blood glucose measurements and in the sampling and analysis of blood.

\section{Can we use the same measures for assessment of glucose control with continuous and intermittent monitoring?}

As noted in the first section of this paper, almost all monitoring of the blood glucose concentration in critically ill patients is by intermittent measurement. Although intermittent measurement is current standard practice, there is no agreed metric for reporting glycemic control and many of the metrics currently reported are affected by the frequency of measurement. Current systems for continuous or automated intermittent monitoring may measure the blood glucose concentration at a frequency varying from every minute to every 15 minutes. Such monitors will not only increase the number of measurements, but will also standardize the frequency of measurements amongst patients monitored with each device. This may allow for a better reporting of glucose control metrics, and if sufficiently accurate may offer a better understanding of the association between those metrics and outcomes. 


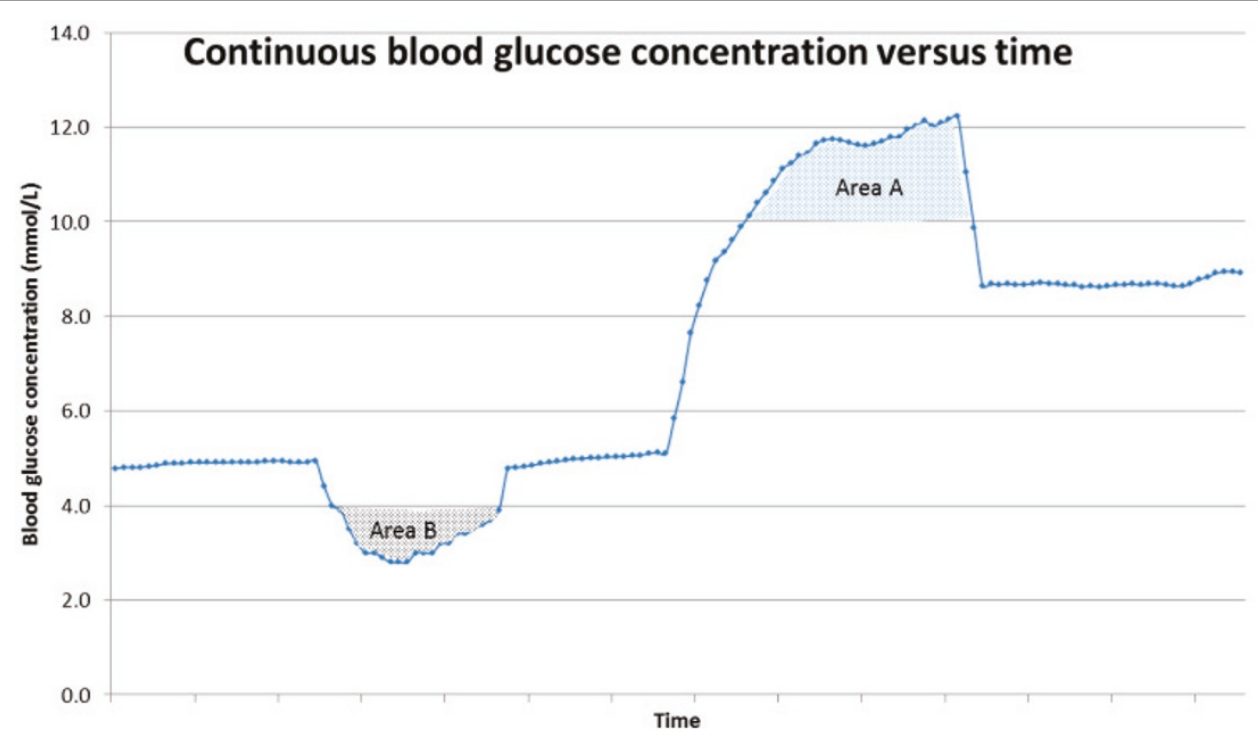

Figure 2. Schematic representation of the performance of continuous blood glucose concentration. Area A, possible measure of hyperglycemia (when defined as blood glucose concentration $>10.0 \mathrm{mmol} / \mathrm{l}$ ). Area B, possible measure of moderate hypoglycemia (when defined as blood glucose concentration $<4.0 \mathrm{mmol} / \mathrm{l})$.

Possible metrics available from continuous glucose monitoring systems include the following (Figure 2 and Table 2):

1. Measures of central tendency:

a. The mean and median blood glucose per patient, per day and during stay in the ICU.

2. Measures of dispersion and variability:

a. The standard deviation and coefficient of variation of blood glucose.

b. Analysis of the rate of change of the blood glucose concentration.

c. The peak blood glucose concentration reached within a set time period after correction of hypoglycemia.

3. Hypoglycemia and lowest blood glucose concentration:

a. The lowest blood glucose concentration recorded.

b. The area above the curve under the target for mild and severe hypoglycemia (Area B in Figure 2, where the target is to keep blood glucose $>4.0 \mathrm{mmol} / \mathrm{l})$.

c. The number of times that a blood glucose concentration below that defining hypoglycemia or severe hypoglycemia is recorded.

d. The duration of time that blood glucose is below the concentration defining hypoglycemia or severe hypoglycemia.

4. Range and exposure measures:

a. The percentage of time the blood glucose concentration is in the target range.

b. The percentage of time the blood glucose concentration is outside a nominated target range. c. The area under the curve above the upper target for hyperglycemia (Area A in Figure 2, where the target is to keep blood glucose $<10.0 \mathrm{mmol} / \mathrm{l}$ ).

$\mathrm{d}$. The area above the curve under the target for mild and severe hypoglycemia (Area B in Figure 2, where the target is to keep blood glucose $>4.0 \mathrm{mmol} / \mathrm{l}$ ).

There are currently few data to guide the choice of appropriate metrics for continuous glucose monitoring. There is a need to define measures that are associated with important patient-centered outcomes such as mortality and major morbidity. The easiest metric to define will be the incidence and severity of hypoglycemia and hyperglycemia; harder to define will be measures of central tendency and dispersion, because these may be influenced by the frequency of measurement.

\section{Comparing glycemic control with continuous versus intermittent measurement of blood glucose}

Comparison of the quality of glycemic control using continuous and intermittent measurement is a crucial first step in determining whether continuous glucose monitoring systems can provide tangible benefit to patients. As frequent knowledge of the blood glucose concentration has the potential to change a patient's management, comparison of the glycemic control achieved with continuous versus intermittent monitoring must be evaluated in a randomized controlled trial with both groups of patients having a continuous monitor but the output from the continuous monitor masked in the control group where blood glucose is managed by intermittent 


\section{Table 2. Metrics to report glycemic control using continuous glucose monitoring systems}

1. Measures of central tendency

a. Mean blood glucose per patient, per day of ICU stay and during whole stay in the ICU

b. For populations of patients, the median and interquartile range of individual patient means should be reported

c. For comparison of glycemic control achieved with intermittent measurement versus continuous measurement, two groups should be studied in a randomized controlled trial, both groups should have blood glucose concentration recorded by a CGM but the output of the CGM should be masked in the control group where blood glucose concentration is managed using intermittent measurement of blood glucose concentration

2. Measures of variability and dispersion

a. Standard deviation and coefficient of variation of blood glucose (for comparison of glycemic control with intermittent measurement, two groups should be studied - both groups should have blood glucose concentration recorded by a CGM but the output of the CGM be masked in the group where the blood glucose concentration is managed using intermittent measurement of blood glucose concentration)

b. Peak blood glucose concentration reached within a set time period after correction of hypoglycemia

c. Analysis of the rate of change of the blood glucose concentration

3. Hypoglycemia and lowest blood glucose concentration

a. Lowest blood glucose concentration recorded per patient

b. Number of times a blood glucose concentration defining hypoglycemia or severe hypoglycemia was recorded

c. Area above the curve under the target for mild and severe hypoglycemia

d. Duration of time with blood glucose below the concentration defining hypoglycemia or severe hypoglycemia

4. Range and exposure measures

a. Percentage of time the blood glucose concentration is in the target range

b. Percentage of time the blood glucose concentration is outside a nominated target range

c. Area under the curve above the upper target for hyperglycemia

d. Area above the curve but under the target for mild and severe hypoglycemia

Desirable performance standards for continuous glucose monitoring systems - the meeting accepted that these were desirable performance standards that might not be currently achievable

There was acceptance but not complete agreement that desirable point accuracy might vary depending on whether CGMs were intended to operate alone and be used to adjust insulin dosing or be used to only as a warning system to advise clinicians when to check blood glucose on a reference system

1. Desirable features with regard to set-up, calibration and integration with standard ICU care

a. $\quad<20$ minutes to set by a nurse, technician, or physician

b. $<10$ minutes required for initial and subsequent calibration

c. $\quad<20$ minutes required for sensor insertion

d. Calibration against a reference standard to maintain point accuracy is required no more frequently than every 8 hours and preferably no more than twice per 24 hours

e. CGM requires few nursing interventions per 24 hours to produce a near-continuous glucose datastream

f. CGM sensor inserted into an arterial or central venous catheter should not adversely affect blood sampling or monitoring of cardiovascular system or increase the frequency with which such monitoring lines occlude

g. Incidence infection, hematoma, tissue ischemia, and/or thromboembolism should not exceed that which occurs in usual clinical practice in the absence of a CGM

2. Desirable reliability

a. CGM should continuously measure glucose and display in real time $>95 \%$ of the time for the duration of time specified in the product label (2 to 7 days)

b. Skips in data acquisition due to system faults or failures should not exceed 30 minutes at a time (data may be missing for other reasons; for example, patient transports)

c. The CGM should have an internal mechanism that prevents the display or reporting of erroneous or spurious data

3. Desirable point accuracy - this should be the same as for intermittent monitors if the CGM is being used alone to guide clinical management and administration of insulin

a. $98 \%$ of readings should be within $12.5 \%$ of a reference standard (or within $0.55 \mathrm{mmol} / \mathrm{l}$ for readings $<5.5 \mathrm{mmol} / \mathrm{l}$; the remaining $2 \%$ of readings should be within $20 \%$ of a reference standard)

b. CGM sensors inserted in arterial and central venous catheters may transiently indicate false low readings when those catheters are flushed with saline or other glucose-free solutions. The CGM should alert the treating clinicians that rapid reductions in the measured blood glucose concentration may be due to flushing of the arterial or central venous catheter

c. CGM sensors inserted in peripheral and central veins may transiently indicate false high readings if venous blood in those veins is contaminated with glucose-containing solutions. The CGM should alert the treating clinicians to rapid increases in the measured blood glucose concentration that may be due to contamination by glucose-containing solutions

4. Rate or trend accuracy - CGMs should be carefully characterized in the intended-use critical care patient population to ensure point accuracy over a wide range of blood glucose rates of change. Rate or trend metrics have not been tested sufficiently to provide definitive guidance

5. Alarms and alerts for hypoglycemia and hyperglycemia alerts

a. Real-time blood glucose concentration and trend data should be displayed at the bedside; with visual and audible alerts and alarms for hypoglycemia, hyperglycemia, and rapid rates of change

b. The CGM data should be sufficiently frequent, reliable, and accurate for alarm algorithms to detect and/or predict hypoglycemia and hyperglycemia with high sensitivity and specificity 
monitoring. This will be the only way to accurately compare the relative effect of continuous versus intermittent monitoring on glycemic control.

\section{What are acceptable performance standards for continuous glucose monitoring systems? (Table 1)}

Continuous or automated intermittent glucose monitoring systems (CGMs) should safely and reliably provide an accurate interstitial fluid or blood glucose measurement every 1 to 15 minutes. They should maintain their accuracy for a period of days and over a wide range of glucose values, rates of change of blood glucose concentration, and patient conditions. Each CGM should demonstrate accuracy in the intended-use critical care population to ensure safety. Point accuracy - the accuracy with which each static blood glucose measurement matches a reference measurement - should be similar to that required of intermittent monitoring systems. Rate or trend accuracy - the accuracy with which the CGM is able to track changes in blood glucose concentration - is a specific feature of continuous glucose monitoring systems and standards have yet to be developed. Standards for rate accuracy may differ if CGMs are intended only to warn clinicians that blood glucose is trending out of range and changes in management are not made until the CGM reading is confirmed by a reference blood glucose measurement. Greater accuracy is required if the CGM readings are to be used to guide therapy, in particular the administration of insulin, without confirmation from a reference blood glucose measurement. CGMs must also demonstrate a lack of interference from substances other than glucose, and a lack of sample contamination by adjacent infusions.

The set-up and calibration of the CGM should be easy to perform in the clinical setting using methods that provide an accurate reference blood glucose measurement; and the CGM should withhold data and alert the clinician when accuracy becomes questionable. The current glucose concentration and trend data should be displayed at the bedside, with visual and audible alerts and alarms for hypoglycemia, hyperglycemia, and rapid rates of change. The CGM data should be sufficiently frequent, reliable, and accurate for alarm algorithms to detect and/or predict hypoglycemia and hyperglycemia with high sensitivity and specificity.

All systems (needle-type CGM sensors inserted into the subcutaneous tissue that provide an interstitial fluid glucose measurement, optical fiber and dialysis catheter CGM sensors inserted directly into the bloodstream, and external CGM sensors attached to a vascular catheter) should ideally provide a glucose measurement that agrees closely with the true blood glucose concentration when recalibrated less than three times per day. Additional studies are required to determine whether adjacent infusions affect CGM performance when attached to a central venous catheter.

Insufficient data currently exist for most systems to demonstrate that they can meet these performance standards in a broad range of critical care settings.

\section{Conclusion}

In view of the lack of standardization in the management, reporting and assessment of glycemic control, we realized that recommendations were needed for the use of different methods to measure the blood glucose concentration and for the report of the performance of insulin treatment. We suggest in this review ways to report the central tendency, dispersion and rate of hypoglycemia when intermittent and continuous blood glucose values are reported. We also suggest minimal performance standards for intermittent and continuous blood glucose monitors when these are used in ICUs. We thereby hope to improve glycemic control in daily clinical practice and to minimize the disparities and to facilitate the interpretation and comparison of clinical trials.

Abbreviations

CGM, continuous or automated intermittent glucose monitoring system.

\section{Competing interests}

SF receives research funding and travel expenses to employer from GluMetrics Inc., provision of equipment for research to employer from Dipylon Medical (Eirus), travel expenses and consulting fees to his employer from Edwards Life Sciences, and provision of equipment for research to employer from Nova Biomedical (StatStrip). JW received project funding from CMA, a company that no longer exists which previously developed a device for intravenous microdialysis for glucose monitoring. JP receives speaker and consultancy fees from Edwards Life Sciences, Medtronic and Optiscan. RH reports having received speaker honoraria from Minimed Medtronic, Lifescan, Eli Lilly, and Novo Nordisk, serving on advisory panel for Animas and Minimed Medtronic, receiving license fees from BBraun and Beckton Dickinson, and having served as a consultant to BBraun and Profil. These companies may have patents/ patent applications in the field. RH has two patent applications in the related field. JJ receives funding to the University for clinical trial, advisory board, and travel to speak at meetings from Medtronic MiniMed, Edwards Lifescience, Echo Therapeutics, GluMetrics. JJ receives funding for laboratory studies from Hospira Inc. and Animas-Johnson \& Johnson. JJ receives funding to the University for advisory board from Diramos. JJ receives funding to the University for consulting from Teleflex. MK receives research funding from American Heart Association, Medtronic MiniMed, GluMetrics, Genentech, Sanofi-Aventis, Gilead, and has served as a consultant for Medtronic MiniMed, GluMetrics, Genentech, Gilead, Boehringer-Ingleheim, and Hoffman-La Roche. JK performs consulting work for Medtronic, Edwards Life Sciences, Roche Diagnostics, and Glysure Ltd. DM received a lecture fee and travel reimbursement from Medtronic during the ESICM 2011 meeting which was paid to the University's Research Fund. MS receives funding to the institution for consulting work from Medtronic Inc., Glysure Ltd., Roche Diagnostics, Edwards Life Sciences, and Optiscan Biomedical. MS's institution receives research funding from Medtronic Inc. and Optiscan Biomedical. MGS performs consulting work for Roche Diagnostics, and Edwards Life Sciences. GVdB receives a consulting fee from Glysure Ltd. TC, TD, IM, RS, and TVH declare they have no conflicts of interest.

\section{Author details}

'The George Institute for Global Health and Royal North Shore Hospital, University of Sydney, St Leonards, Sydney, NSW 2065, Australia. ${ }^{2}$ Department of Anestesiology \& Intensive Care Medicine, Karolinska University Hospital, Solnavägen 1, 17177 Solna, Sweden. ${ }^{3}$ Department of Intensive Care, Erasme University Hospital, 808 route de Lennik, B-1070 Brussels, Belgium. ${ }^{4}$ Institute 
of Biomedical Engineering, Imperial College, South Kensington Campus, Exhibition Rd, London SW7 2AZ, UK. ${ }^{5}$ GIGA - Cardiovascular Sciences, University of Liege, Institute of Physics, B5, Allee du 6 aout, 17, 4000 Liege, Belgium. ${ }^{6}$ nstitute of Metabolic Science, University of Cambridge Metabolic Research Laboratories, Level 4, Institute of Metabolic Science, Box 289, Addenbrooke's Hospital, Hills Rd, Cambridge CB2 0QQ, UK. ${ }^{7}$ Jefferson Artificial Pancreas Center \& Anesthesiology Program for Translational Research, Department of Anesthesiology, Jefferson Medical College of Thomas Jefferson University, 1020 Walnut Street, Philadelphia, PA 19107, USA. ${ }^{8}$ Saint-Luke's Mid America Heart Institute, University of Missouri - Kansas City, 4401 Wornal Road, Kansas City, MO 64111, USA. ' Division of Critical Care, Stamford Hospital and Columbia University College of Physicians and Surgeons, 30 Shelburne Road, Stamford, CT 06904, USA. ${ }^{10}$ Department of Anaesthesia and Intensive Care Medicine, Queen Elizabeth Hospital Birmingham, Queen Elizabeth Medical Centre, Birmingham, B15 2TH, UK. "'Department of Intensive Care Medicine, University Hospitals Leuven, Herestraat 49, B-3000 Leuven, Belgium. ${ }^{12}$ Department of Intensive Care Medicine, Academic Medical Center at the University of Amsterdam, Meibergdreef 9, 1105 AZ Amsterdam, The Netherlands. ${ }^{13}$ Department of Pathology and Immunology, Division of Laboratory and Genomic Medicine, Washington University School of Medicine, 660 South Euclid Avenue, St Louis, MO 63110, USA. ${ }^{14}$ Isala Klinieken, Department of Clinical Chemistry, Groot Wezenland 20, 8011 JW Zwolle, The Netherlands. ${ }^{15}$ Department of Electrical Engineering (ESAT-SCD), Katholieke Universiteit Leuven, Leuven, Belgium

Published: 14 June 2013

\section{References}

1. Van den Berghe G, Wouters P, Weekers F, Verwaest C, Bruyninckx F, Schetz M, Vlasselaers D, Ferdinande P, Lauwers P, Bouillon R: Intensive insulin therapy in critically ill patients. N Eng/ J Med 2001, 345:1359-1367.

2. Kanji S, Buffie J, Hutton B, Bunting PS, Singh A, McDonald K, Fergusson D, McIntyre LA, Hebert PC: Reliability of point-of-care testing for glucose measurement in critically ill adults. Crit Care Med 2005, 33:2778-2785.

3. Mackenzie I, Whitehouse T, Nightingale P: The metrics of glycaemic control in critical care. Intensive Care Med 2011, 37:435-443.

4. Hoedemaekers CW, Klein Gunnewiek JM, Prinsen MA, Willems JL, Van der Hoeven JG: Accuracy of bedside glucose measurement from three glucometers in critically ill patients. Crit Care Med 2008, 36:3062-3066.

5. Scott MG, Bruns DE, Boyd JC, Sacks DB: Tight glucose control in the intensive care unit: are glucose meters up to the task? Clin Chem 2009, 55:18-20.

6. Van den Berghe G, Wilmer A, Hermans G Meersseman W, Wouters PJ, Milants I, Van Wijngaerden E, Bobbaers H, Bouillon R: Intensive insulin therapy in the medical ICU. N Engl J Med 2006, 354:449-461.

7. Brunkhorst FM, Engel C, Bloos F, Meier-Hellmann A, Ragaller M, Weiler N, Moerer O, Gruendling M, Oppert M, Grond S, Olthoff D, Jaschinski U, John S, Rossaint R, Welte T, Schaefer M, Kern P, Kuhnt E, Kiehntopf M, Hartog C, Natanson C, Loeffler M, Reinhart K; German Competence Network Sepsis (SepNet): Intensive insulin therapy and pentastarch resuscitation in severe sepsis. N Engl J Med 2008, 358:125-139.

8. Preiser JC, Devos P, Ruiz-Santana S, Mélot C, Annane D, Groeneveld J, lapichino G, Leverve X, Nitenberg G, Singer P, Wernerman J, Joannidis M, Stecher A, Chioléro R: A prospective randomised multi-centre controlled trial on tight glucose control by intensive insulin therapy in adult intensive care units: the Glucontrol study. Intensive Care Med 2009, 35:1738-1748.

9. NICE-SUGAR Study Investigators: Intensive versus conventional glucose control in critically ill patients. N Engl J Med 2009, 360:1346-1349.

10. Taylor $\mathrm{JH}$, Beilman GJ: Hyperglycemia in the intensive care unit: no longer just a marker of illness severity. Surg Infect (Larchmt) 2005, 6:233-245.
11. Egi M, Bellomo R, Stachowski E French CJ, Hart GK, Taori G, Hegarty C, Bailey M: Hypoglycemia and outcome in critically ill patients. Mayo Clin Proc 2010 85:217-224.

12. Egi M, Bellomo R, Stachowski E, French CJ, Hart G: Variability of blood glucose concentration and short-term mortality in critically ill patients. Anesthesiology 2006, 105:244-252.

13. Dungan K, Chapman J, Braithwaite SS, Buse J: Glucose measurement: confounding issues in setting targets for inpatient management. Diabetes Care 2007, 30:403-409.

14. Eslami S, Taherzadeh Z, Schultz MJ, Abu-Hanna A: Glucose variability measures and their effect on mortality: a systematic review. Intensive Care Med 2011, 37:583-593.

15. Van Herpe T, Mesotten D, Wouters PJ, Herbots J, Voets E, Buyens J, De Moor B, Van den Berghe G: LOGIC - insulin algorithm-guided versus nurse-directed blood glucose control during critical illness: the LOGIC-1 single-center randomized, controlled clinical trial. Diabetes Care 2013, 36:188-194.

16. Finkielman JD, Oyen $\sqcup$, Afessa B: Agreement between bedside blood and plasma glucose measurement in the ICU setting. Chest 2005, 127:1749-1751.

17. Lyon ME, Baskin LB, Braakman S, Presti S, Dubois J, Shirey T: Interference studies with two hospital-grade and two home-grade glucose meters. Diabetes Technol Ther 2009, 11:641-647.

18. Boyd JC, Bruns DE: Monte Carlo simulation in establishing analytical quality requirements for clinical laboratory tests meeting clinical needs. Methods Enzymol 2009, 467:411-433.

19. Breton MD, Kovatchev BP: Impact of blood glucose self-monitoring errors on glucose variability, risk for hypoglycemia, and average glucose control in type 1 diabetes: an in silico study. J Diabetes Sci Technol 2010, 4:562-570.

20. Clarke WL, Cox D, Gonder-Frederick LA, Carter W, Pohl SL: Evaluating clinical accuracy of systems for self-monitoring of blood glucose. Diabetes Care 1987, 10:622-628.

21. Brunner R, Kitzberger R, Miehsler W, Herkner H, Madl C, Holzinger U: Accuracy and reliability of a subcutaneous continuous glucose-monitoring system in critically ill patients. Crit Care Med 2011, 39:659-664.

22. US Department of Health and Human Services: Clinical Laboratory Improvement Amendments of 1988; final rules and notice. 42 CFR Part 493. Federal Register 1992, 57:7188-7288.

23. Jansen RT, Slingerland RJ: SKML - quality mark for point-of-care test (POCT) glucose meters and glucose meters for home-use. Clin Chem Lab Med 2010, 48:1021-1027.

24. Royal College of Pathologists of Australia Quality Assurance Program: Revision of Allowable Limits of Performance; 2010. [http://www.westgard.com/rcpaaustralasian-quality-requirements.htm]

25. Karon BS, Gandhi GY, Nuttall GA Bryant SC, Schaff HV, McMahon MM, Santrach PJ: Accuracy of Roche Accu-Chek Inform whole blood capillary, arterial, and venous glucose values in patients receiving intensive intravenous insulin therapy after cardiac surgery. Am J Clin Pathol 2007, 127:919-926.

26. Sinha S, Jayaram R, Hargreaves CG: Fatal neuroglycopaenia after accidental use of a glucose $5 \%$ solution in a peripheral arterial cannula flush system. Anaesthesia 2007, 62:615-620.

27. Oliver NS, Toumazou C, Cass AEG, Johnston DG: Glucose sensors: a review of current and emerging technology. Diabetic Med 2009, 26:197-210.

\section{doi:10.1186/cc12537}

Cite this article as: Finfer S, et al:: Clinical review: Consensus recommendations on measurement of blood glucose and reporting glycemic control in critically ill adults. Critical Care 2013, 17:229. 


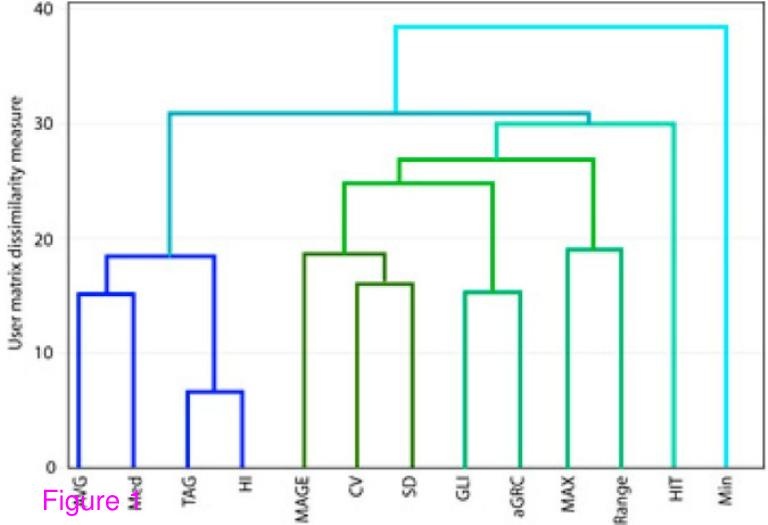


${ }^{14.0} \quad$ Continuous blood glucose concentration versus time

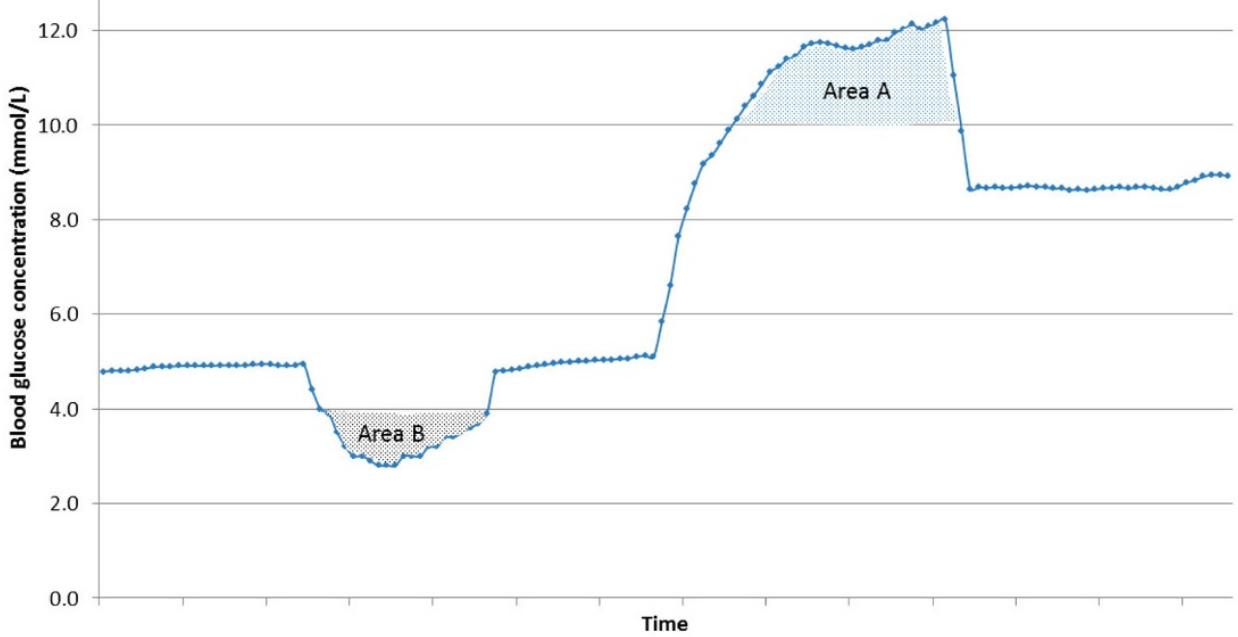

